\title{
Structure, spectra and variability of some GPS radio sources
}

\author{
X. Liu $^{1, \star}$, H. -G. Song ${ }^{1}$, and L. Cui ${ }^{1,2}$ \\ 1 National Astronomical Observatories/Urumqi observatory, CAS, 40-5 South Beijing Rd, Urumqi 830011, PR China \\ ${ }^{2}$ Graduate University of the Chinese Academy of Sciences, Beijing 100049, PR China
}

Received 2008, accepted 2008

Key words galaxies: nuclei - quasars: general - radio continuum: galaxies

\begin{abstract}
We report the results of multifrequency-VLBI observations of GHz-Peaked-Spectrum (GPS) radio sources. The VLBI structure and component spectra of some GPS sources are presented. Our VLBI results show that about $80 \%$ of the GPS galaxies exhibit a compact double or CSO-like structure, while the GPS quasars tend to show a core-jet. The component spectra of the GPS galaxies are often steep/convex, and the core has a flat spectrum but it is usually hidden or weak. In addition, we studied the variability of GPS sources by comparing new flux density measures, acquired with the Urumqi $25 \mathrm{~m}$ telescope at $4.85 \mathrm{GHz}$, with previous $87 \mathrm{~GB}$ data. The results show that $44 \%$ of the GPS quasars varied higher than $10 \%$ in passed 20 years, while the fraction is only $12 \%$ for the GPS galaxies meaning that the GPS quasars are much more variable than GPS galaxies. In total, $25 \%$ of GPS sources show $>10 \%$ variability at $4.85 \mathrm{GHz}$ in our sample.
\end{abstract}

(c) 2008 WILEY-VCH Verlag GmbH \& Co. KGaA, Weinheim

\section{Introduction}

GHz-Peaked-Spectrum (GPS) radio sources are powerful $\left(P_{1.4 \mathrm{GHz}} \geq 10^{25} \mathrm{~W} \mathrm{~Hz}^{-1}\right)$ and compact $(\leq 1 \mathrm{kpc})$, characterized by a convex radio spectrum that peaks between 0.3-10 GHz (observer's frame), and they represent a significant fraction $(\sim 10 \%)$ of the bright radio source population (O'Dea 1998). Only a few percent of them have weak extended emission (Stanghellini et al. 2005). A couple of GPS sources are also identified as Compact Symmetric Objects (CSOs, e.g Owsianik \& Conway 1998). It is believed that GPS-small sizes are most likely due to their youth $(<$ $10^{4}$ years) than to a dense confining medium (Murgia et al. 1999; O'Dea et al. 2005). In this scenario, these sources will evolve into large radio sources $(>15 \mathrm{kpc})$, some of them would evolve into FRII radio sources (Fanti et al. 1995, Snellen et al. 2000). However, the GPS phenomenon is not completely understood, e.g. the distribution of sizes/ages, structure/spectra, and variability of GPS sources. We have carried out EVN (European VLBI Network) observations of 19 GPS sources, 15 of them are from the Parkes halfJansky sample (Snellen et al. 2002) with declination $>-5^{\circ}$ and not observed with VLBI before (except 2121-014 and 2322-040 we observed), four others are from our previous observation list which observed with the EVN at 2.3/8.4 $\mathrm{GHz}$ and/or $5 \mathrm{GHz}$ (Xiang et al. 2005, 2006), the $1.6 \mathrm{GHz}$ observation will further provide information on the source structure and spectra. Furthermore, we have carried out flux density observations with the Urumqi $25 \mathrm{~m}$ telescope in order to study GPS flux density variability, searching for a different behavior between galaxies and quasars.

\footnotetext{
* Corresponding author: e-mail: liux@uao.ac.cn
}

\section{VLBI structure and spectra of GPS sources}

The $1.6 \mathrm{GHz}$ VLBI observation was carried out on 2006 March 3 using the MK5 recording system with a bandwidth of $32 \mathrm{MHz}$ and sample rate of $256 \mathrm{Mbps}$. The EVN antennae in this experiment were Effelsberg, Westerbork, Jodrell, Medicina, Noto, Onsala, Torun, Hartebeesthoek, Urumqi, and Shanghai. Snapshot observations of 19 sources (Table 1) in total of 24 hours were made. OQ208 was observed as a calibrator. The Astronomical Image Processing System (AIPS) has been used for editing, a-priori calibration, fringe fitting, self-calibration, and imaging of the data.

The results show that 12 out of 15 sources from Snellen (2002) sample exhibit compact doubles, 2 sources exhibit core-jet structure, and $\mathrm{J} 1648+0242$ is totally resolved out. For 4 others sources in Table 1,3 show compact doubles, one shows a core-jet. The last six sources in Table 1 were also observed at $2.3 / 8.4 \mathrm{GHz}$ and/or $5 \mathrm{GHz}$ before this 1.6 $\mathrm{GHz}$ run. We summarize the VLBI structure of 19 sources in Table 1. Our results show that about $80 \%$ of the GPS galaxies exhibit a compact double or CSO like structure. The quasar J1203+0414 tends to show a core-jet. We have identified 4 CSOs from the sources which observed at 4 frequencies, according to their symmetric and steep spectrum of mini lobes. In the following we show the images and spectra of two sources as examples.

\subsection{PKS 0914+114}

The source has a GPS spectrum (Stanghellini et al. 1998), recently it has been optically identified as an empty field (Labiano et al. 2007). In the 1.6 GHz VLBI image, it shows a core component $\mathrm{A}$, one-side jet $\mathrm{B}$ and two lobes $\mathrm{C}, \mathrm{E}$ (Liu et al. 2007). Considering the source structure at $1.6 \mathrm{GHz}$, we 
Table 1 The GPS sources. Columns (1),(2) source names; (3) optical identification (gl: galaxy, Q: quasar, EF: empty field); (4) optical magnitude; (5) redshift (de Vries et al. 2007, those with * are photometric estimated by Tinti et al. 2005); (6) linear scale factor $\mathrm{pc} / \mathrm{mas}\left[H_{0}=71 \mathrm{kms}^{-1} \mathrm{Mpc}^{-1}\right.$ and $q_{0}=0.5$ have been assumed]; (7) maximum angular size from the observation; (8) maximum linear size; (9) VLBI structure (cd: compact double, cj: core-jet, n: no detection); (10) low frequency spectral index; (11) higher frequency spectral index; (12) turnover frequency; (13) peak flux density; (14) references for the spectral information, 1 Snellen et al. 2002, 2 de Vries et al. 1997, 3 Stanghellini et al. 1998, where $S \propto \nu^{-\alpha}$.

\begin{tabular}{|c|c|c|c|c|c|c|c|c|c|c|c|c|c|}
\hline $\begin{array}{c}1 \\
\text { Name }\end{array}$ & $\begin{array}{c}2 \\
\text { other }\end{array}$ & $\begin{array}{c}3 \\
i d\end{array}$ & $\begin{array}{c}4 \\
m_{R}\end{array}$ & $\begin{array}{l}5 \\
z\end{array}$ & $\begin{array}{c}6 \\
p c / m a s\end{array}$ & $\begin{array}{c}7 \\
\theta \\
\text { mas }\end{array}$ & $\begin{array}{c}8 \\
L \\
\mathrm{pc}\end{array}$ & $\begin{array}{c}9 \\
v l b i\end{array}$ & $\begin{array}{l}10 \\
\alpha_{l}\end{array}$ & $\begin{array}{l}11 \\
\alpha_{h}\end{array}$ & $\begin{array}{c}12 \\
\nu_{m} \\
\mathrm{GHz}\end{array}$ & $\begin{array}{c}13 \\
S_{m} \\
\mathrm{Jy}\end{array}$ & $\begin{array}{c}14 \\
\text { ref }\end{array}$ \\
\hline J0210+0419 & В $0208+040$ & $\mathrm{gl}$ & $>24.1$ & $1.5^{*}$ & 6.1 & 90 & & $\mathrm{~cd}$ & & 0.80 & 0.4 & 1.3 & 1 \\
\hline $\mathrm{J} 0323+0534$ & $4 C+05.14$ & $\mathrm{gl}$ & 19.2 & 0.1785 & 2.7 & 180 & 490 & $\mathrm{~cd}$ & & 0.85 & 0.4 & 7.1 & 1 \\
\hline J0433-0229 & $4 C-02.17$ & $\mathrm{gl}$ & 19.1 & 0.530 & 5.1 & 80 & 408 & $\mathrm{~cd}$ & & 0.52 & 0.4 & 3.0 & 1 \\
\hline J0913+1454 & B0910+151 & $\mathrm{gl}$ & 22.9 & $0.47^{*}$ & 4.9 & 80 & & $\mathrm{~cd}$ & & 0.75 & 0.6 & 1.1 & 1 \\
\hline $\mathrm{J} 1057+0012$ & B1054+004 & $\mathrm{gl}$ & 22.3 & $0.65^{*}$ & 5.5 & $80 ?$ & & cj & & 0.67 & 0.4 & 1.6 & 1 \\
\hline $\mathrm{J} 1109+1043$ & B1107+109 & $\mathrm{gl}$ & 22.6 & $0.55^{*}$ & 5.2 & 60 & & $\mathrm{~cd}$ & & 0.94 & 0.5 & 2.4 & 1 \\
\hline $\mathrm{J} 1135-0021$ & $4 C-00.45$ & $\mathrm{gl}$ & 21.9 & 0.975 & 6.0 & 120 & 720 & $\mathrm{~cd}$ & & 0.78 & 0.4 & 2.9 & 1 \\
\hline $\mathrm{J} 1203+0414$ & B1200+045 & $\mathrm{Q}$ & 18.8 & 1.221 & 6.1 & 75 & 458 & cj & & 0.45 & 0.4 & 1.4 & 1 \\
\hline $\mathrm{J} 1352+0232$ & B1349+027 & $\mathrm{gl}$ & 20.0 & 0.607 & 5.4 & 170 & 918 & $\mathrm{~cd}$ & & 0.58 & 0.4 & 2.0 & 1 \\
\hline $\mathrm{J} 1352+1107$ & $4 C+11.46$ & $\mathrm{gl}$ & 21.0 & 0.891 & 5.9 & 50 & 295 & $\mathrm{~cd}$ & & 1.03 & 0.4 & 3.6 & 1 \\
\hline $\mathrm{J} 1600-0037$ & B1557-004 & $\mathrm{gl}$ & & & & 50 & & $\mathrm{~cd} / \mathrm{cj}$ & & 1.17 & 1.0 & 1.2 & 1 \\
\hline $\mathrm{J} 1648+0242$ & $4 C+02.43$ & $\mathrm{gl}$ & 22.1 & 0.824 & 5.8 & & & $\mathrm{n}$ & & & 0.4 & 3.4 & 1 \\
\hline $\mathrm{J} 2058+0540$ & $4 C+05.78$ & $\mathrm{gl}$ & 23.4 & 1.381 & 6.1 & 160 & 970 & $\mathrm{~cd}$ & & 0.95 & 0.4 & 3.1 & 1 \\
\hline J2123-0112 & B2121-014 & $\mathrm{gl}$ & 23.3 & 1.158 & 6.1 & 80 & 488 & cso & -0.56 & 0.75 & 0.4 & 2.0 & 1 \\
\hline $\mathrm{J} 2325-0344$ & B2322-040 & $\mathrm{gl}$ & 23.5 & 1.509 & 6.0 & 75 & 450 & cso & -0.42 & 0.75 & 1.4 & 1.2 & 1 \\
\hline $\mathrm{J} 0917+1113$ & B0914+114 & $\mathrm{EF}$ & & & & 190 & & cso & -0.1 & 1.6 & 0.3 & 2.3 & 3 \\
\hline $\mathrm{J} 1753+2750$ & B1751+278 & $\mathrm{gl}$ & 21.7 & $0.86^{*}$ & 5.9 & 50 & & cj & -0.27 & 0.57 & 1.4 & 0.6 & 2 \\
\hline $\mathrm{J} 1826+2708$ & B1824+271 & $\mathrm{gl}$ & 22.9 & & & 45 & & cso & -0.39 & 0.75 & 1.0 & 0.4 & 2 \\
\hline $\mathrm{J} 2325+7917$ & B $2323+790$ & $\mathrm{gl}$ & $19.5 \mathrm{~V}$ & & & 32 & & $\mathrm{~cd}$ & -0.3 & 0.75 & 1.4 & 1.2 & 2 \\
\hline
\end{tabular}

Table 2 Flux densities (mJy) of VLBI components, the value with $*$ is an upper limit. The error is $\sim 10 \%$ of flux density.

\begin{tabular}{crrrrr}
\hline Source & comp & $S_{1.6}$ & $S_{2.3}$ & $S_{5}$ & $S_{8.4}$ \\
\hline $0914+114$ & $\mathrm{~A}$ & 50 & 38 & 52 & 19 \\
& $\mathrm{~B}$ & 65 & 14 & 3 & $1^{*}$ \\
& $\mathrm{C}$ & 360 & 186 & 31 & 6 \\
$2121-014$ & $\mathrm{E}$ & 40 & 17 & 10 & $1^{*}$ \\
& $\mathrm{~A}$ & 594 & 353 & 196 & 113 \\
& $\mathrm{~B}$ & $10^{*}$ & 120 & 14 & $4^{*}$ \\
& $\mathrm{C}$ & 415 & 237 & 107 & 29 \\
\hline
\end{tabular}

have re-processed the data at $2.3,5,8.4 \mathrm{GHz}$ with careful calibration and 'clean' in AIPS, the component $\mathrm{E}$ has been restored at $2.3,5 \mathrm{GHz}$, and component $\mathrm{C}$ is restored at 8.4 GHz, as shown in Fig. 1, Fig. 2, Fig. 3.

The component spectra (Fig. 4) from the VLBI images at $1.6,2.3,5$, and $8.4 \mathrm{GHz}$, show that the core A has a flatter spectrum than other components. The data are listed in Table 2. Based on its structure and spectra, we classify this GPS source as a CSO.

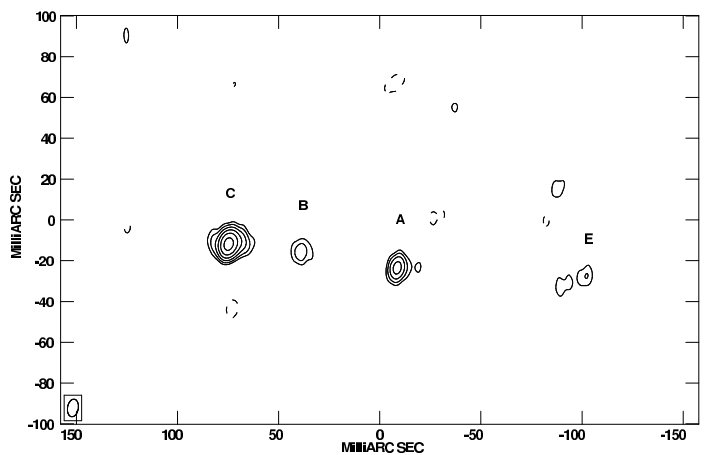

Fig. $10914+114$ at $2.3 \mathrm{GHz}$, the restoring beam is $10.4 \times$ 7.3 mas with PA $-15.5^{\circ}$. The peak is $164 \mathrm{mJy} / \mathrm{beam}$, the first contour is $2 \mathrm{mJy} / \mathrm{beam}$. The contour levels here and below increase by a factor of 2 .

\section{$2.2 \quad$ PKS 2121-014}

This GPS source is hosted by a galaxy at a redshift of 1.158 . Using the VLBI results at 1.6, 2.3, 5, and $8.4 \mathrm{GHz}$ we obtained the spectra for each component (Fig. (5), the two lobes A, C show steep spectra, in which we have reprocessed the 8.4 GHz data as shown in Fig. 6 with careful calibration 


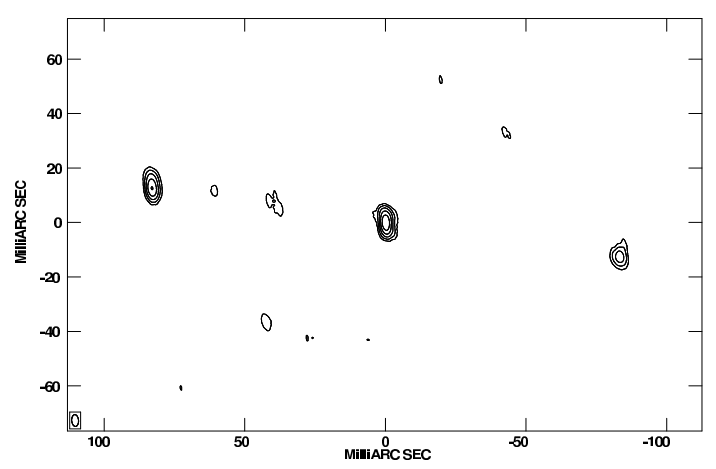

Fig. $20914+114$ at $5 \mathrm{GHz}$, the restoring beam is $4.3 \times$ 2.4 mas with PA $3.9^{\circ}$. The peak is $30.7 \mathrm{mJy} /$ beam, the first contour is $1 \mathrm{mJy} / \mathrm{beam}$.

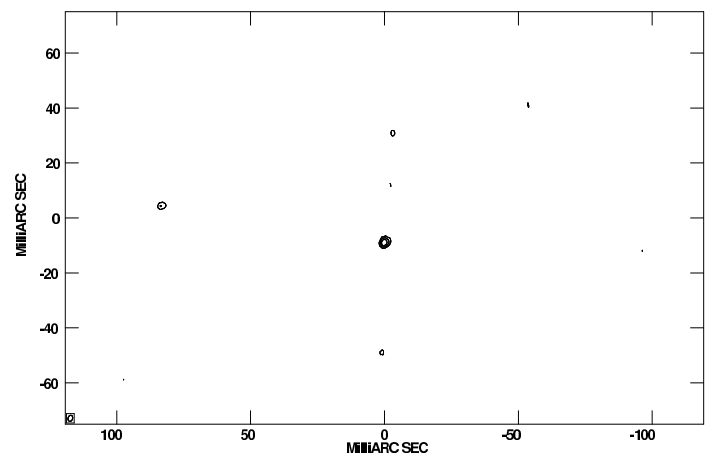

Fig. $30914+114$ at $8.4 \mathrm{GHz}$, the restoring beam is $2.2 \times$ 1.7 mas with PA $-18.5^{\circ}$. The peak is $11.1 \mathrm{mJy} / \mathrm{beam}$, the first contour is $1.5 \mathrm{mJy} / \mathrm{beam}$.

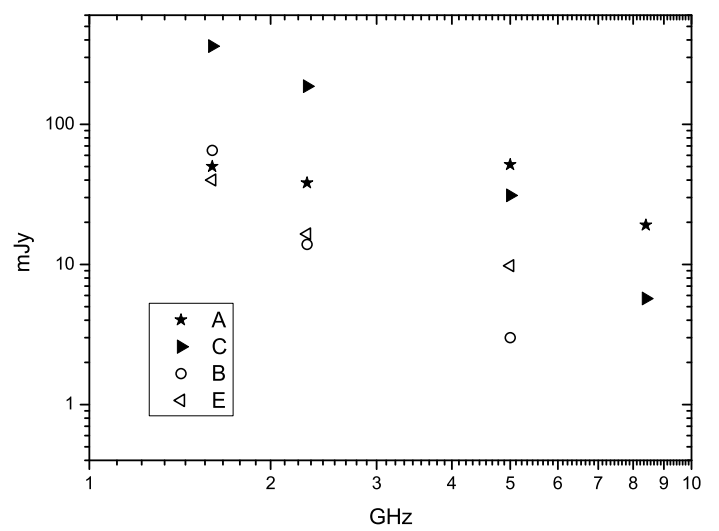

Fig. 4 Component spectra of 0914+114 at 1.6, 2.3, 5, and $8.4 \mathrm{GHz}$. Total flux density peaks at $0.3 \mathrm{GHz}$.

and 'clean' in AIPS. As discussed in Xiang et al. (2006), the two components $\mathrm{A}, \mathrm{C}$ in the $8.4 \mathrm{GHz}$ image (Xiang et al. 2005) might be swapped due to worse phase calibration, so the new image Fig. 6 is a correction. The component B as seen at 2.3, $5 \mathrm{GHz}$ (Xiang et al. 2005, 2006) was not detected in this $1.6 \mathrm{GHz}$ observation, assuming an upper limit of $10 \mathrm{mJy}$ set by $3 \sigma$ in the image, it has an inverted spectrum which is a sign of a jet or probably an absorbed core. Based on its double structure and steep spectra of lobes, we classify this GPS source as a CSO.

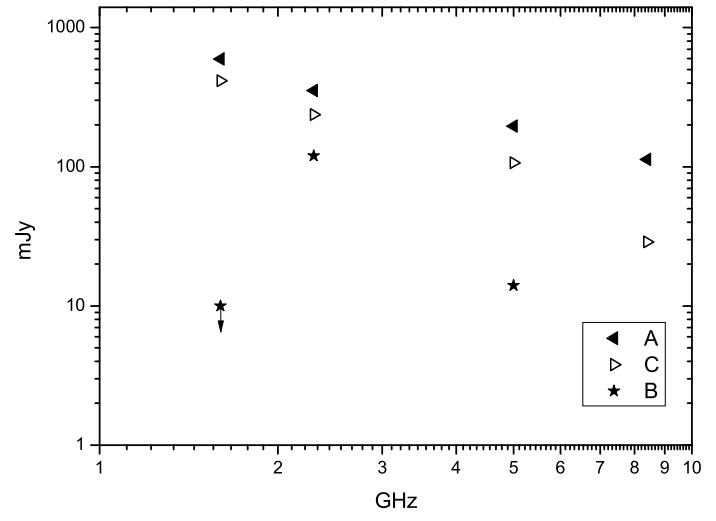

Fig. 5 Component spectra of 2121-014 at 1.6, 2.3, 5, and $8.4 \mathrm{GHz}$. Total flux density peaks at $0.4 \mathrm{GHz}$.

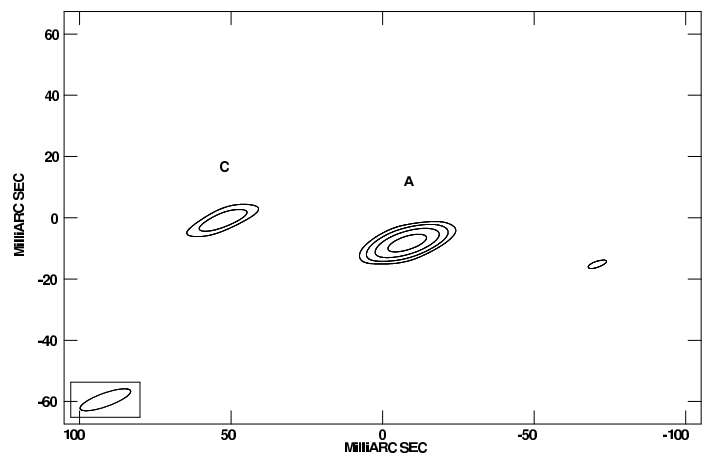

Fig. $62121-014$ at $8.4 \mathrm{GHz}$, the restoring beam is $17.6 \times$ 4.4 mas with PA $-70.3^{\circ}$. The peak is $82.7 \mathrm{mJy} / \mathrm{beam}$, the first contour is $7 \mathrm{mJy} / \mathrm{beam}$.

\section{Flux variability of GPS sources}

It is reported that a lot of GPS sources show long term variability (Torniainen et al. 2005) especially at high radio frequency. In order to check this also at centimeter wavelength, in 2007 July, we carried out flux density measurements at $4.85 \mathrm{GHz}$ for a large sample of $172 \mathrm{GPS}$ sources edited by Labiano et al. (2007). By comparing these data with the $87 \mathrm{~GB}$ and PMN data at the same frequency, we obtained the flux variations of the GPS sources between the two epoches. Because low source declination and/or bad Gaussian fits, only flux densities of 121 sources were obtained.

Flux densities were determined with 'cross - scans' in azimuth and elevation, fourfold in each coordinate. This enables us to check the pointing offsets in both coordinates. A Gaussian fit was performed on each sub-scan, and after applying a correction for pointing offsets the amplitudes of both AZ and EL were averaged. Then we correct the measurements for the antenna gain, and finally the flux densities were scaled to $7.55 \mathrm{Jy}$ of $3 \mathrm{C} 286$.

As in Fig.7 only 9 out of 73 GPS galaxies (12\%) show flux variation higher than $10 \%(>2 \sigma)$; at the same time 21 out of 48 quasars $(44 \%)$ show variability higher than $10 \%$ $(>2 \sigma)$. The result indicates that GPS quasars are much more variable than GPS galaxies. The variable sources are 
X. Liu et al.: Structure, spectra and variability of GPS sources

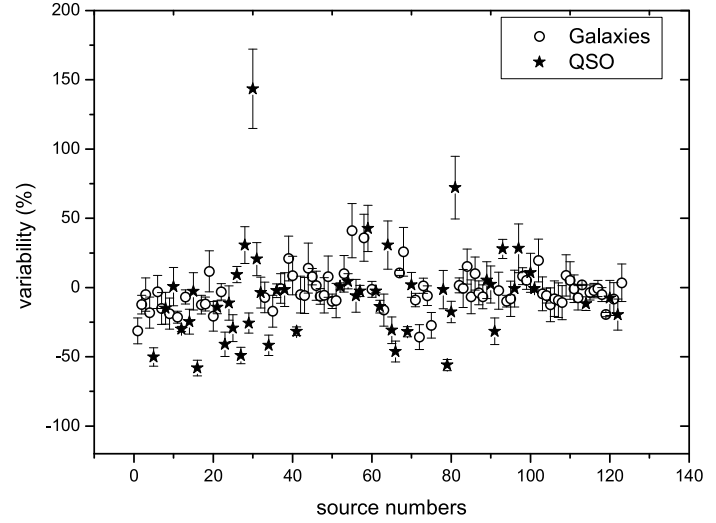

Fig. 7 Flux variation (\%) at $4.85 \mathrm{GHz}$, by comparing the data measured in 2007 at Urumqi telescope with 87GB/PMN data for 121 GPS sources from Labiano et al. sample.

listed in Table 3, columns 1 to 6 are source name (1950), optical identification, $4.85 \mathrm{GHz}$ flux density measured at Urumqi, $4.85 \mathrm{GHz}$ flux density from $87 \mathrm{~GB}$ or PMN data, and flux variation and the references (1: Gregory \& Condon 1991; 2: Griffith et al. 1994; 3: Becker et al. 1991). Sources in Table 1 are also in Labiano sample, of them two sources show variability, the core-jet source $1054+004$ shows a variation of $(11.4 \pm 3.6) \%$, and $1557-004$ shows a variation of (13.4 \pm 6.9$) \%$ just at $2 \sigma$ level.

\section{Summary and discussion}

Our VLBI results show that $80 \%$ of the GPS galaxies exhibit a compact double or CSO-like structure. However, for 13 sources which were only observed at $1.6 \mathrm{GHz}$ further VLBI observations are needed for classifying their structure type in detail. Combining the 1.6, 2.3/8.4, and $5 \mathrm{GHz}$ VLBI observations for other 6 sources, 4 CSOs are classified according to their steep spectrum of double lobes. The GPS galaxies are dominated by jet/lobe emission, with a hidden or weak core (it was not detected because of sensitivity in most of our sources), suggesting they are at large angles to the line of sight. While GPS quasars may be at moderate angles to the line of sight (Stanghellini et al. 2001).

Only $12 \%$ of GPS galaxies show flux variation higher than $10 \%$, while $44 \%$ of GPS quasars show variability higher than $10 \%$ in passed 20 years. The result indicates that most GPS galaxies are stable in flux density, and the GPS quasars are much more variable than GPS galaxies. The variable GPS quasars often show compact core-jet, e.g. the quasar $0642+449$ which has 140\% flux variation in Fig.77is an extremely compact core-dominated one at a redshift of 3.4. Torniainen et al. (2005) found that 54\% of GPS sources show flux variability in long term (with fractional variability index $>3$ ). In our result, $25 \%$ of GPS sources show $>10 \%$ variation, the difference may be due to different samples and the definition of variability, we simply compared the flux densities in two epoches.
Table 3 Flux density variation $(>2 \sigma)$ of the GPS sources.

\begin{tabular}{ccrrrr}
\hline Source & id & $S_{u r}$ & $87 \mathrm{~GB} / \mathrm{PMN}$ & variation & ref \\
\hline $0000+212$ & gl & $242 \pm 6$ & $352 \pm 47$ & $-31.3 \pm 9.3$ & 1 \\
$0039+230$ & $\mathrm{Q}$ & $820 \pm 6$ & $1645 \pm 220$ & $-50.2 \pm 6.7$ & 1 \\
$0207-224$ & gl & $486 \pm 1$ & $618 \pm 34$ & $-21.4 \pm 4.3$ & 2 \\
$0237-233$ & $\mathrm{Q}$ & $2541 \pm 5$ & $3630 \pm 99$ & $-30 \pm 1.9$ & 2 \\
$0248+430$ & $\mathrm{Q}$ & $1065 \pm 6$ & $1414 \pm 169$ & $-24.7 \pm 9$ & 1 \\
$0354+231$ & $\mathrm{Q}$ & $137 \pm 2$ & $327 \pm 44$ & $-58.1 \pm 5.7$ & 1 \\
$0405-280$ & gl & $487 \pm 4$ & $553 \pm 31$ & $-11.9 \pm 5.0$ & 2 \\
$0434-188$ & $\mathrm{Q}$ & $934 \pm 4$ & $1089 \pm 58$ & $-14.2 \pm 4.6$ & 2 \\
$0457+024$ & $\mathrm{Q}$ & $996 \pm 5$ & $1689 \pm 253$ & $-41.0 \pm 8.8$ & 1 \\
$0507+179$ & $\mathrm{Q}$ & $549 \pm 5$ & $777 \pm 106$ & $-29.3 \pm 9.7$ & 1 \\
$0621+446$ & $\mathrm{Q}$ & $188 \pm 3$ & $369 \pm 43$ & $-49.1 \pm 6.0$ & 1 \\
$0636+680$ & $\mathrm{Q}$ & $371 \pm 17$ & $499 \pm 43$ & $-25.7 \pm 7.3$ & 1 \\
$0642+449$ & $\mathrm{Q}$ & $2900 \pm 7$ & $1191 \pm 140$ & $143.5 \pm 28.6$ & 1 \\
$0711+356$ & $\mathrm{Q}$ & $525 \pm 2$ & $901 \pm 113$ & $-41.7 \pm 7.3$ & 1 \\
$0858-279$ & $\mathrm{Q}$ & $1516 \pm 12$ & $2216 \pm 99$ & $-31.6 \pm 3.1$ & 2 \\
$1054+004$ & gl & $351 \pm 6$ & $396 \pm 23$ & $-11.4 \pm 3.6$ & 2 \\
$1200+045$ & gl & $721 \pm 5$ & $511 \pm 71$ & $41.1 \pm 19.6$ & 1 \\
$1315+415$ & gl & $242 \pm 3$ & $178 \pm 22$ & $36.0 \pm 16.9$ & 1 \\
$1333+459$ & $\mathrm{Q}$ & $853 \pm 7$ & $598 \pm 70$ & $42.6 \pm 16.7$ & 1 \\
$1354-174$ & $\mathrm{Q}$ & $870 \pm 5$ & $1009 \pm 54$ & $-13.8 \pm 4.6$ & 2 \\
$1427+109$ & $\mathrm{Q}$ & $855 \pm 5$ & $1236 \pm 171$ & $-30.8 \pm 9.6$ & 1 \\
$1502+036$ & $\mathrm{Q}$ & $533 \pm 3$ & $991 \pm 138$ & $-46.2 \pm 7.5$ & 1 \\
$1519-273$ & $\mathrm{Q}$ & $1250 \pm 18$ & $1835 \pm 96$ & $-31.9 \pm 3.7$ & 2 \\
$1543+005$ & gl & $839 \pm 5$ & $1306 \pm 182$ & $-35.7 \pm 9.0$ & 1 \\
$1600+335$ & gl & $1492 \pm 7$ & $2051 \pm 262$ & $-27.3 \pm 9.3$ & 1 \\
$1622+665$ & g1 & $229 \pm 6$ & $520 \pm 46$ & $-55.9 \pm 4.1$ & 1 \\
$1645+635$ & $\mathrm{Q}$ & $366 \pm 6$ & $444 \pm 41$ & $-17.6 \pm 7.7$ & 1 \\
$1648+015$ & $\mathrm{Q}$ & $787 \pm 5$ & $457 \pm 60$ & $72.2 \pm 22.6$ & 3 \\
$2019+050$ & $\mathrm{Q}$ & $468 \pm 6$ & $684 \pm 95$ & $-31.6 \pm 9.5$ & 1 \\
$2126-158$ & $\mathrm{Q}$ & $1517 \pm 12$ & $1186 \pm 63$ & $27.9 \pm 6.9$ & 2 \\
\hline & & & & &
\end{tabular}

GPS galaxies which show comparable double lobes can be interpreted as type-2 AGN in the framework of unified scheme. GPS quasars, which show a core-jet structure and variability, could be mostly type-1 AGN.

Acknowledgements. This work was supported by the National Natural Science Foundation of China (NSFC) under grant No.10773019 and the 973 Program of China under grant No.2009CB824800.

\section{References}

Becker, R.H., White, R.L., Edwards, A.L.: 1991, ApJS 75, 1 de Vries, N., Snellen, I.A.G., et al.: 2007, A\&A 464, 879 de Vries, W.H., Barthel, P.D., O’Dea, C.P.: 1997, A\&A 321, 105 Fanti, C., Fanti, R., Dallacasa, D., et al.: 1995, A\&A 302, 317 Gregory, P.C., Condon, J.J.: 1991, ApJS 75, 1011 Griffith, M.R., Wright, A.E., et al.: 1994, ApJS 90, 179 Labiano, A, Barthel, P.D., O’Dea, C.P., et al.: 2007, A\&A 463, 97 Liu, X., Cui, L., Luo, W.F., et al.: 2007, A\&A 470, 97 Murgia, M., Fanti, C., Fanti, R., et al.: 1999, A\&A 345, 769 O’Dea, C.P., Gallimore, J., et al.: 2005, AJ 129, 610

O’Dea, C.P.: 1998, PASP 110, 493

Owsianik I., Conway J.E.: 1998, A\&A 337, 69

Snellen, I.A.G., Lehnert, M.D., et al.: 2002, MNRAS 337, 981

Snellen, I.A.G., Schilizzi, R.T., et al.: 2000, MNRAS 319, 445

Stanghellini, C., Dallacasa, D., et al.: 2001, A\&A 377, 377

Stanghellini, C., O’Dea, C.P., et al.: 1998, A\&AS 131, 303 
Tinti, S., Dallacasa, D., de Zotti, G., et al.: 2005, A\&A 432, 31

Torniainen, I., Tornikoski, M., et al.: 2005, A\&A 435, 839

Xiang, L., Dallacasa, D., Cassaro, P., et al.: 2005, A\&A 434, 123

Xiang, L., Reynolds, C., Strom, R.G., et al.: 2006, A\&A 454, 729 